UG-FT-67/96

hep-ph/9607313

July 1996

\title{
Numerical diagonalization of fermion mass matrices
}

\author{
J. A. Aguilar-Saavedra \\ Departamento de Física Teórica y del Cosmos \\ Universidad de Granada \\ 18071 Granada, Spain
}

\begin{abstract}
The diagonalization of general mass matrices is a more delicate problem when eigenvalue degeneracies exist. In this case, often overlooked in the literature, some difficulties arise related to the freedom in the choice of basis in degenerate subspaces. Here two simple algorithms are developed to deal with quark and neutrino mass matrices with arbitrary degeneracies.
\end{abstract}

\section{Introduction}

In a field theory with fermionic fields $\psi_{i}$, the mass sector of the Lagrangian can be written as

$$
-\mathcal{L}_{\text {mass }}=\bar{\psi}_{i} M_{i j} \psi_{j}+\text { h.c. }
$$

The structure of the mass matrix $M$ is constrained by the symmetries of the Lagrangian: charge conservation, baryon and lepton number conservation, etc. We will consider the quark and neutrino sectors of the Standard Model with $N$ generations of quarks; $n_{L}$ left-handed and $n_{R}$ right-handed neutrinos. The mass terms in the quark sector are

$$
-\mathcal{L}_{\text {mass }}=\bar{u}_{L i}^{0} M_{i j}^{u} u_{R j}^{0}+\bar{d}_{L i}^{0} M_{i j}^{d} d_{R j}^{0}+\text { h.c. },
$$

where $u_{L, R}^{0}\left(d_{L, R}^{0}\right)$ are the left and right-handed components of the up (down) quark fields, and $i, j=1, \ldots, N$ label the different generations of up (down) quarks. The $N$-dimensional mass matrices $M^{u, d}$ are complex and arbitrary. In practice it is convenient to work in a quark basis in which $M^{u, d}$ are diagonal. We make an appropriate change of weak quark basis

$$
u_{L i}=U_{L i j}^{u} u_{L j}^{0}, \quad u_{R i}=U_{R i j}^{u} u_{R j}^{0}, \quad d_{L i}=U_{L i j}^{d} d_{L j}^{0}, \quad d_{R i}=U_{R i j}^{d} d_{R j}^{0},
$$

such that the matrices $D^{u} \equiv U_{L}^{u} M^{u} U_{R}^{u \dagger}, D^{d} \equiv U_{L}^{d} M^{d} U_{R}^{d \dagger}$ are diagonal, and rewrite the mass term as

$$
-\mathcal{L}_{\text {mass }}=\bar{u}_{L i} D_{i j}^{u} u_{R j}+\bar{d}_{L i} D_{i j}^{d} d_{R j}+\text { h.c. }
$$


Of course this change of basis may affect other terms in the Lagrangian, but we will not discuss this here. Thus what we denote here as 'diagonalization' is really a biunitary transformation

$$
U_{1} M U_{2}^{\dagger}=D
$$

such that $D$ is diagonal (we can also make the matrix elements of $D$ real and positive). The matrices $U_{1,2}$ can be calculated [1] noting that (5) and $U_{2} M^{\dagger} U_{1}^{\dagger}=D$ imply

$$
U_{1} M M^{\dagger} U_{1}^{\dagger}=D^{2}, \quad U_{2} M^{\dagger} M U_{2}^{\dagger}=D^{2}
$$

However, this does not determine $U_{1,2}$ uniquely if $D$ has degenerate diagonal elements, and this arbitrariness may cause that $D$ is no longer diagonal: There are matrices $U_{1,2}$ satisfying (6) that do not necessarily satisfy (5). For instance, let us consider

$$
M=\left(\begin{array}{ll}
0 & 1 \\
1 & 0
\end{array}\right)
$$

If we try to calculate $U_{1,2}$ by using Eqs. (6), we find $M M^{\dagger}=M^{\dagger} M=I$, so $U_{1}=U_{2}=I$ and $U_{1} M U_{2}^{\dagger}$ is not diagonal. This simple example shows us that we need great care to deal with the case of degenerate eigenvalues.

The mass terms in the neutrino sector are [2]

$$
-\mathcal{L}_{\text {mass }}=\frac{1}{2} \bar{\psi}_{L} M_{\nu} \psi_{R}+\text { h.c. }
$$

with

$$
\psi_{L}=\left(\begin{array}{c}
\nu_{L} \\
\left(\nu^{c}\right)_{L}
\end{array}\right), \quad \psi_{R}=\left(\begin{array}{c}
\left(\nu^{c}\right)_{R} \\
\nu_{R}
\end{array}\right),
$$

and $M_{\nu}$ a complex $\left(n_{L}+n_{R}\right) \times\left(n_{L}+n_{R}\right)$ symmetric matrix. Under a change of basis

$$
N_{L}=U \psi_{L}, \quad N_{R}=U^{*} \psi_{R}
$$

the mass term can be written as

$$
-\mathcal{L}_{\text {mass }}=\frac{1}{2} \bar{N}_{L} D_{\nu} N_{R}+\text { h.c. }
$$

with $D_{\nu}=U M_{\nu} U^{T}$ a diagonal matrix with real positive elements (it will be shown below that any complex symmetric matrix $M_{\nu}$ can be 'diagonalized' with a congruent transformation of this type. A proof for the nondegenerate case can be found in Ref. [3]). If $D$ is nondegenerate we can find the matrix $U$ by diagonalizing $M_{\nu} M_{\nu}^{\dagger}$, but if some degeneracies are present we can get incorrect results. 
In this paper we obtain two algorithms for the diagonalization of complex arbitrary and complex symmetric matrices, respectively, and we write a package Diagon for their use with Mathematica 沺. This package makes use of Eigensystem to calculate eigenvalues and eigenvectors of hermitian and unitary matrices.

\section{Algorithms}

We will first write an algorithm to diagonalize a complex arbitrary matrix by a biunitary transformation and then use it to obtain a second one that performs the diagonalization of a complex symmetric matrix by a congruent transformation. Note that we could use the first algorithm to diagonalize a complex symmetric matrix with two unitary matrices, but in general the transformation would not be congruent.

Let $M$ be a complex arbitrary matrix. It is a well-known fact that we can find two unitary matrices $U_{1}, U_{2}$ such that $U_{1} M U_{2}^{\dagger}=D$ is a diagonal matrix with real positive elements. We will assume for simplicity that $D$ has diagonal elements $d_{i}$ with multiplicity $m_{i}$ and ordered from lower to higher values. Let $V_{1}, V_{2}$ be two matrices that diagonalize $M M^{\dagger}$ and $M^{\dagger} M$ respectively, $V_{1} M M^{\dagger} V_{1}^{\dagger}=D^{2}, V_{2} M^{\dagger} M V_{2}^{\dagger}=D^{2}$. It can be proven that $V_{i}=K_{i} U_{i}$, where $K_{i}$ are block-diagonal unitary matrices that commute with $D$

$$
D=\left(\begin{array}{cccc}
d_{0} I_{m_{0}} & & & \\
& d_{1} I_{m_{1}} & & \\
& & \ddots & \\
& & & d_{k} I_{m_{k}}
\end{array}\right) \Rightarrow K_{i}=\left(\begin{array}{cccc}
K_{i}^{(0)} & & & \\
& K_{i}^{(1)} & & \\
& & \ddots & \\
& & & K_{i}^{(k)}
\end{array}\right)
$$

(the dimension of the blocks $K_{i}^{(j)}$ is $m_{j}$ ). Then,

$$
V_{1} M V_{2}^{\dagger}=K_{1} D K_{2}^{\dagger} \equiv D_{B}
$$

The matrix in the right-hand side of Eq. (13) is a block-diagonal matrix. Each block is an unitary matrix times the corresponding eigenvalue of $D: D_{B}^{(j)}=d_{j} K_{1}^{(j)} K_{2}^{(j) \dagger}$. Furthermore, from $D_{B} D_{B}^{\dagger}=D^{2}$ we can compute the eigenvalues $d_{i}$ and defining $\lambda_{i}=1 / d_{i}$ (if $d_{0}=0$, we take $\lambda_{0}=1$ and $\left.D_{B}^{(0)}=I_{m_{0}}\right)$,

$$
\Lambda=\operatorname{diag}(\overbrace{\lambda_{0}, \ldots, \lambda_{0}}^{m_{0}}, \ldots, \overbrace{\lambda_{k}, \ldots, \lambda_{k}}^{m_{k}})
$$

\footnotetext{
${ }^{1}$ Although Mathematica has a built-in function SingularValues that performs the diagonalization of an arbitrary matrix by a biunitary transformation, it neglects all zero eigenvalues so it is useless for our purpose.
} 
we find that $U_{1} \equiv V_{1}, U_{2} \equiv \Lambda D_{B} V_{2}$ fulfill $U_{1} M U_{2}^{\dagger}=D$.

The diagonalization of a complex symmetric matrix $M_{s}$ by a congruent transformation is somewhat more involved. Let $U_{1}, U_{2}$ be two unitary matrices satisfying $U_{1} M_{s} U_{2}^{\dagger}=D$, with $D$ diagonal with real positive elements (we can calculate $U_{1,2}$ as it is shown above). As $M_{s}$ is symmetric, $U_{2}^{*} M_{s} U_{1}^{T}=D$ and these equations imply $U_{2}=K U_{1}^{*}$, with $K$ a block-diagonal unitary matrix that commutes with $D$ (analogous to $K_{i}$ in Eq. (12)). Then,

$$
U_{1} M_{s} U_{1}^{T}=D K
$$

We now want to decompose $K$ into two unitary symmetric matrices which commute with $D$. The simplest way to show it is to see that $K$ is a symmetric matrix (all the blocks $K^{(j)}$ are symmetric except possibly the block corresponding to $d_{0}=0$, but in this case we can redefine $U_{2}$ without changing $U_{1}$ and take $K^{(0)}=I$ ). A complex unitary symmetric matrix $K$ can always be written as $K=e^{i S}$, with $S$ a real symmetric matrix [5], and $[K, D]=0$ implies $[S, D]=0$, so we can rewrite Eq. (15) as

$$
e^{-i \frac{S}{2}} U_{1} M_{s} U_{1}^{T} e^{-i \frac{S}{2}}=D
$$

However, we need a method to separate the matrix $K$ into two symmetric pieces. First we diagonalize $K$ with a real orthogonal matrix $O: K=O^{T} K_{d} O$. It can always be donef but we must check that the subroutine we use to calculate eigenvectors gives real results if it is possible (the built-in functions Eigenvectors and Eigensystem do it). We conventionally define the square root of $K$ as $K^{1 / 2}=O^{T} K_{d}^{1 / 2} O$, with the eigenvectors in the matrix $O$ conveniently ordered so that $K^{1 / 2}$ commutes with $D$. Finally, $U=\left(K^{1 / 2}\right)^{\dagger} U_{1}$ fulfills $U M_{s} U^{T}=D$.

\section{Examples}

Before we present two examples to illustrate the use of the package Diagon, it is important to notice that when the matrix $m$ has degenerate eigenvalues, the set of eigenvectors calculated with $u=E$ igenvectors [m] is not necessarily an unitary matrix, and the product $u$. Transpose [Conjugate [u] ] may have large off-diagonal elements $\epsilon \sim 10^{-1}$. This is not a roundoff error (typically of order $O\left(10^{-16}\right)$ ) as we show in the example below with an exact diagonalization. We use DiagonalizeH, a subroutine to diagonalize hermitian matrices that uses Eigensystem and the Gram-Schmidt method of orthonormalization in each degenerate subspace to yield orthonormal eigenvectors. The following example will illustrate this. The numerical results have been obtained running Mathematica 2.2.2 for Linux.

\footnotetext{
${ }^{2}$ Note that $S$ is a real symmetric matrix
} 
We write a previously calculated hermitian matrix $m$ with eigenvalues $(0,0,1,1)$ and diagonalize it. Note that DiagonalizeH does not return the eigenvectors but their complex conjugate.

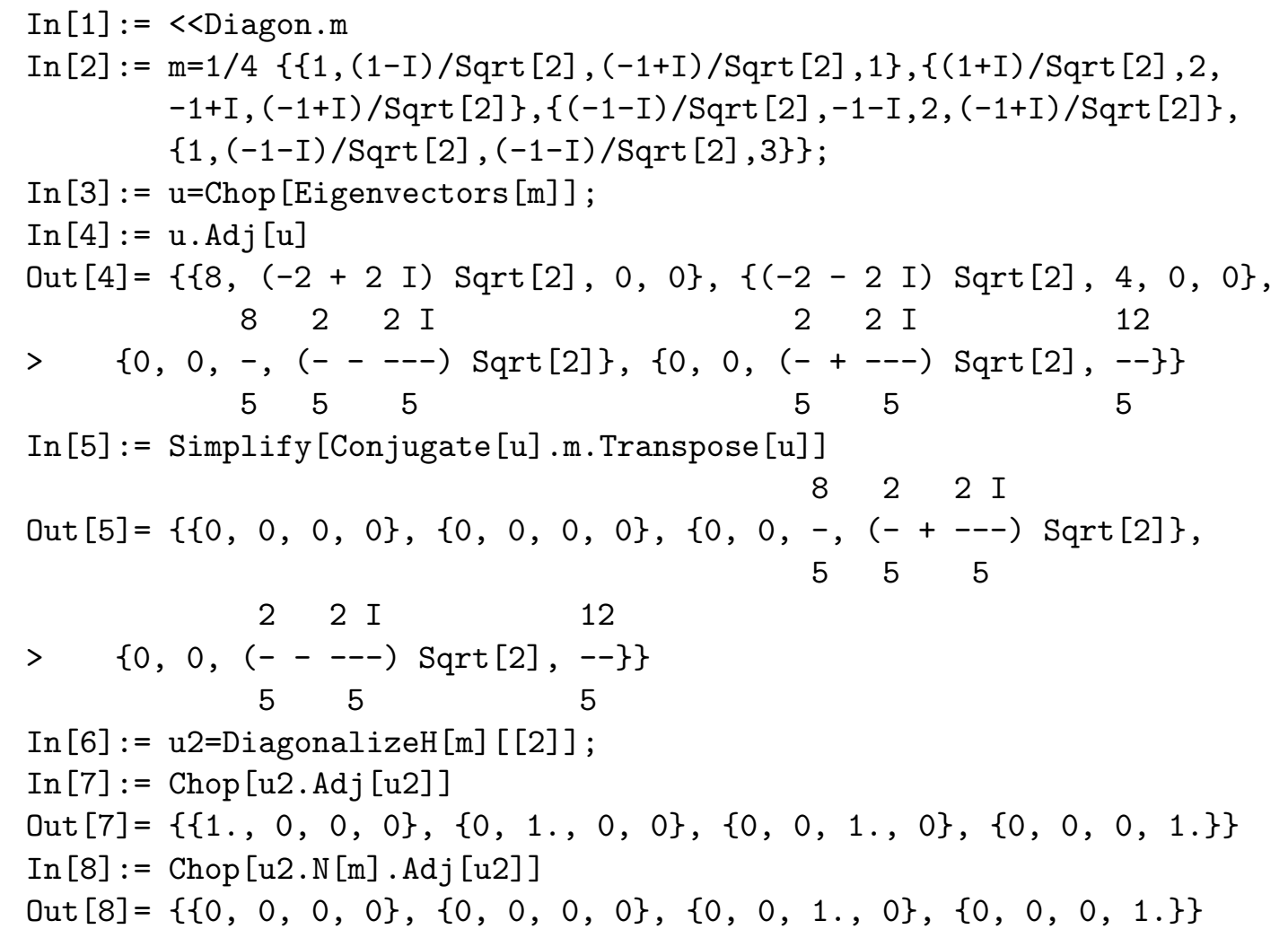

The package Diagon (see the Appendix for a complete description) contains the subroutines DiagonalizeH, Diagonalize and DiagonalizeS. It has been tested more than 100 times on Mathematica 2.2.2 for Linux and Mathematica 2.2 for Windows. We have used the instructions listed below with different sets of eigenvalues, obtaining always correct results. First we load the package and define the matrices $m$ and $m s$ to test Diagonalize and DiagonalizeS respectively. We use 16-digit precision in the input matrices.

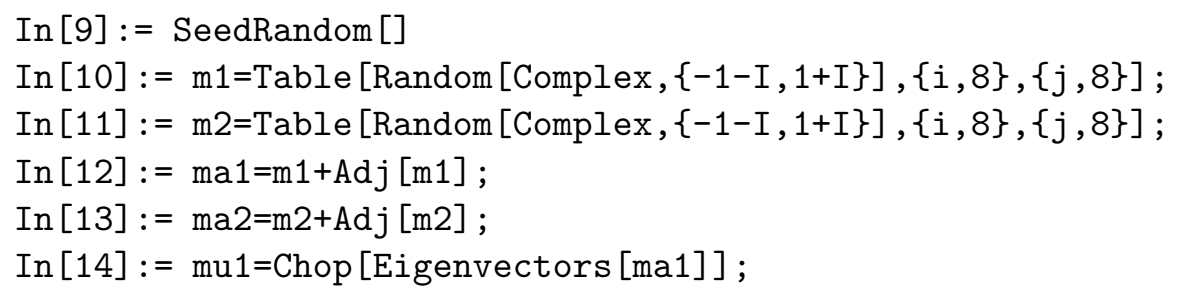




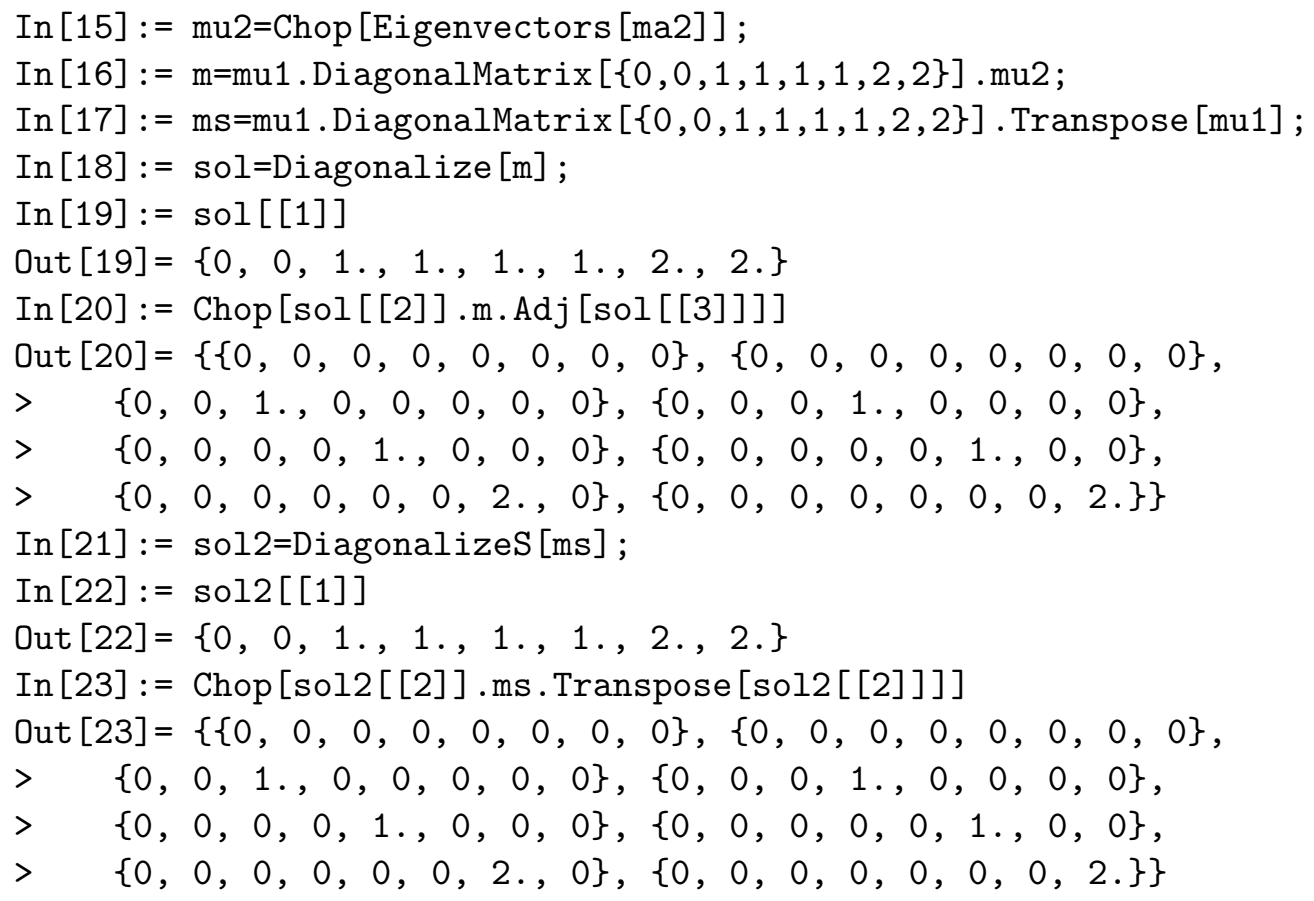

\section{Acknowledgements}

I wish to thank F. del Aguila and M. Masip for revising the manuscript. I also thank J. Jiménez for useful comments. This work was partially supported by CICYT under contract AEN94-0936, by the Junta de Andalucía and by the European Union under contract CHRX-CT92-0004. 


\section{A Appendix}

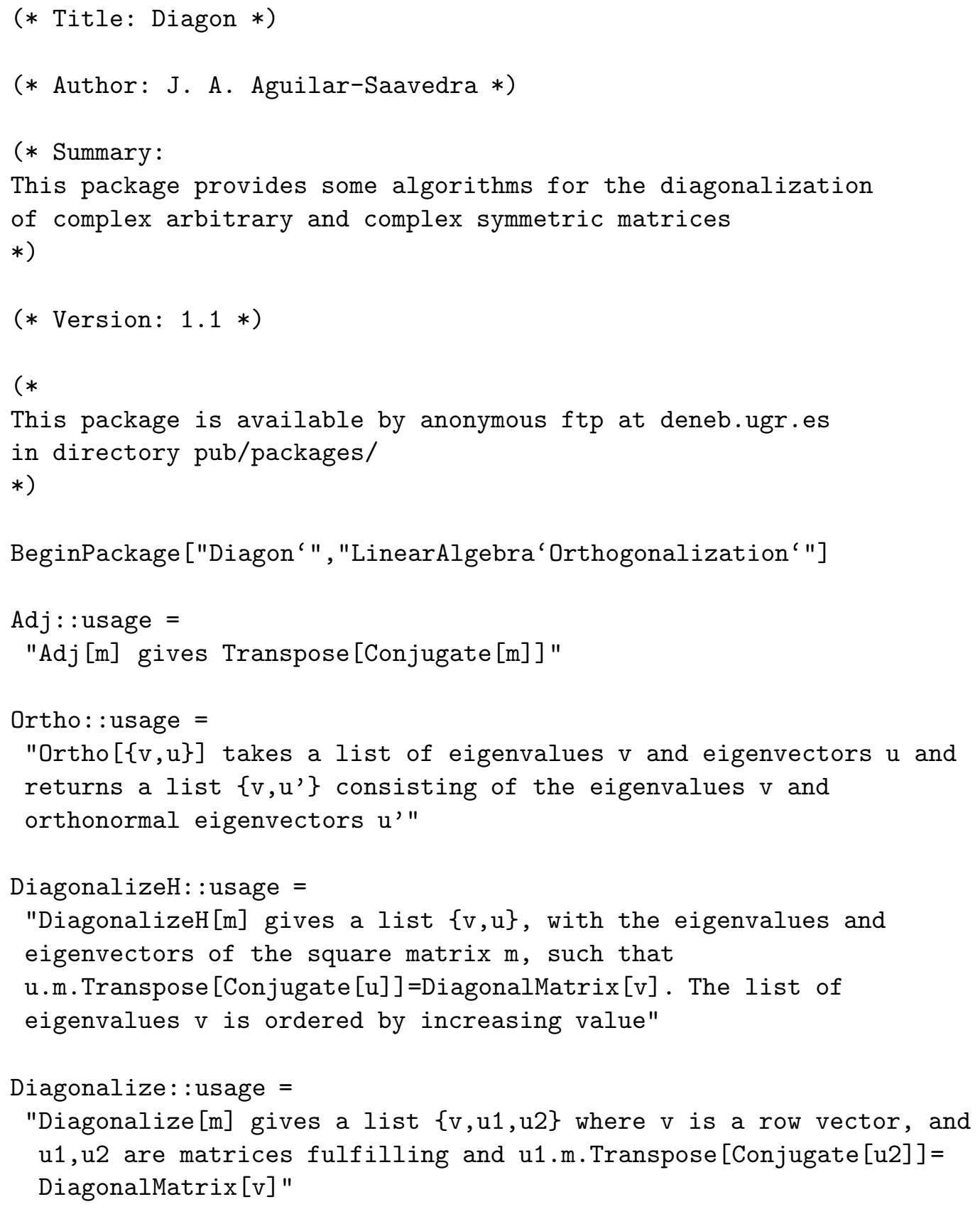




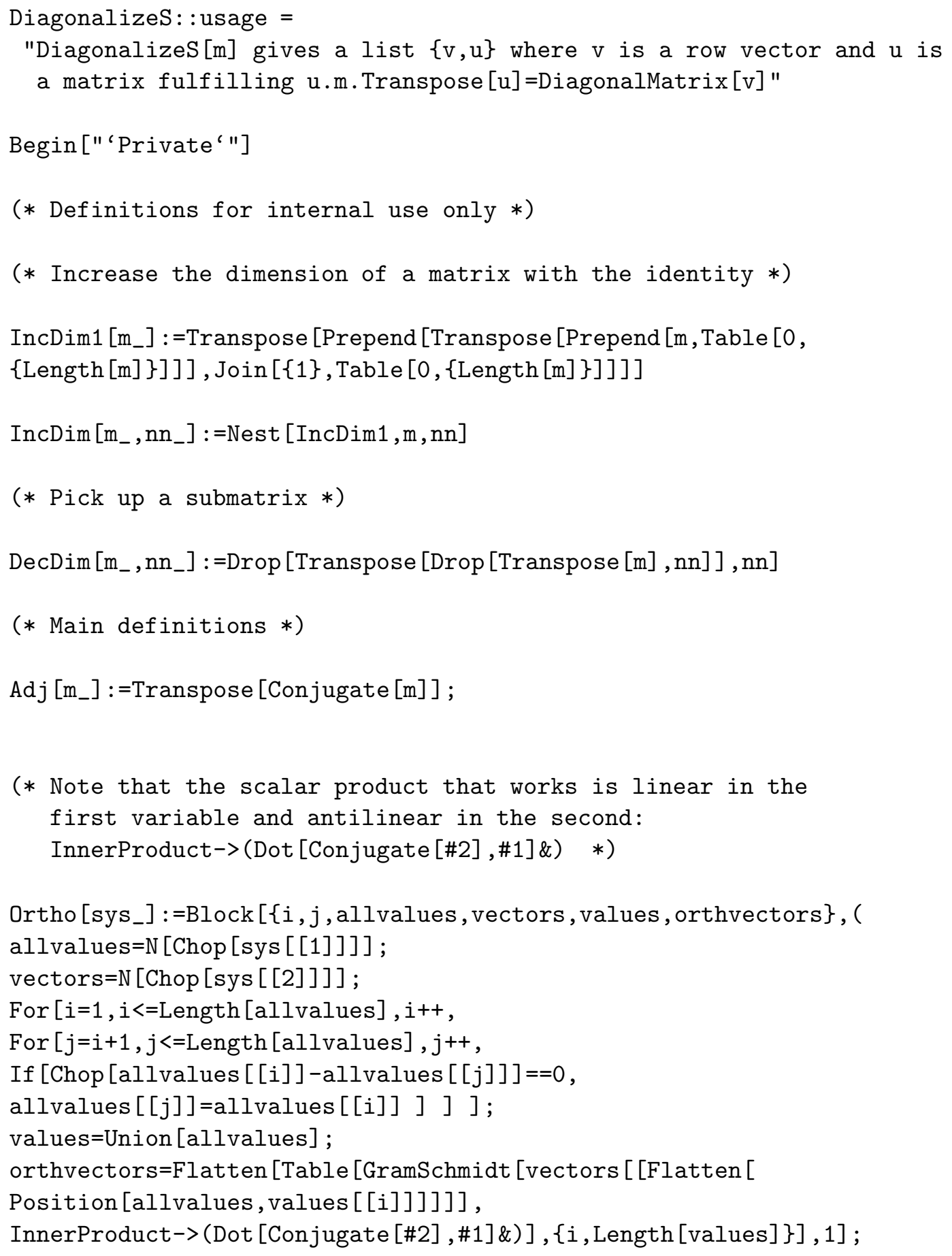




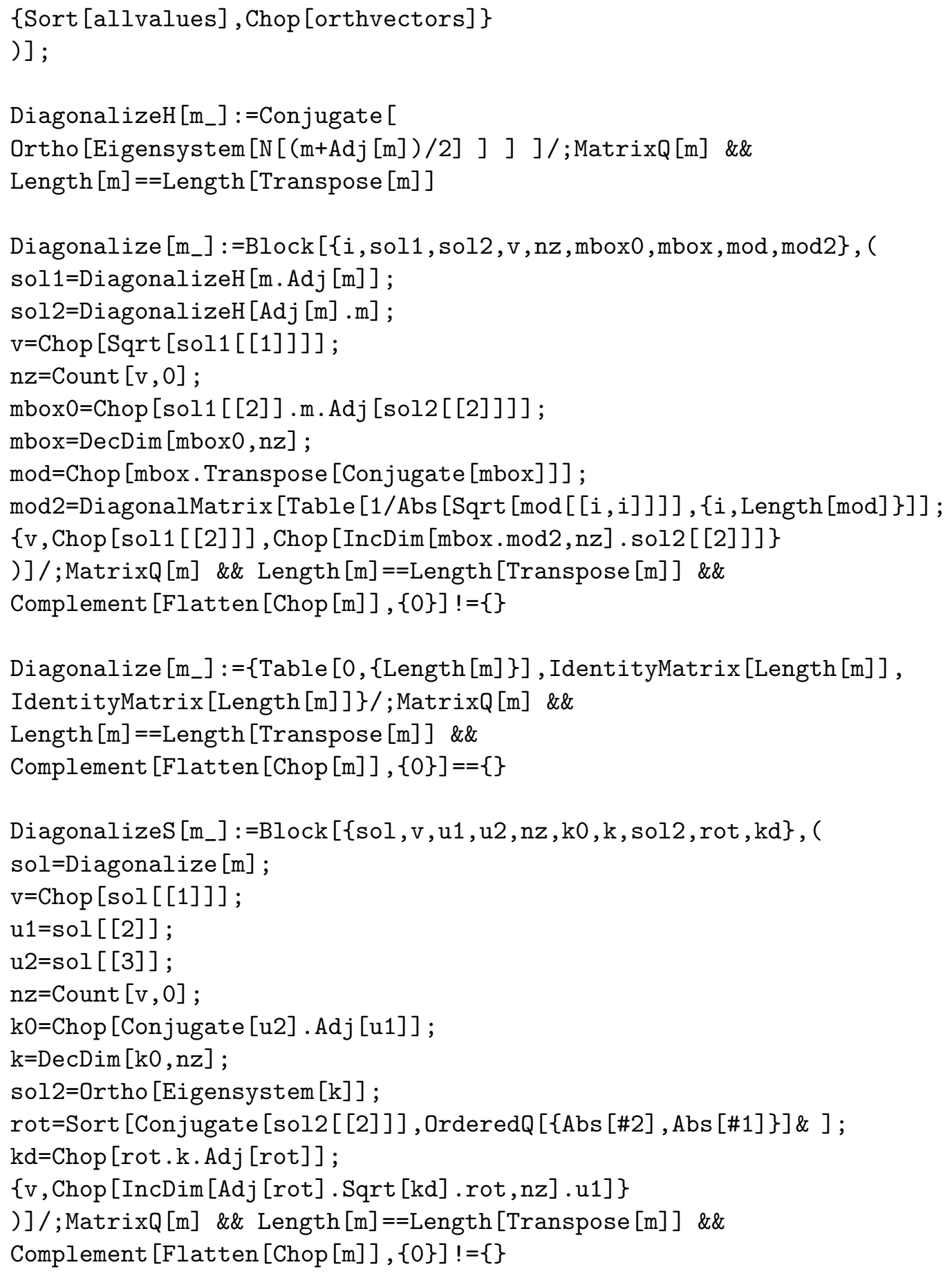


DiagonalizeS $\left[\mathrm{m}_{-}\right]:=\{\mathrm{Table}[0,\{$ Length $[\mathrm{m}]\}]$,

IdentityMatrix [Length [m]]\}/;MatrixQ [m] \&\&

Length $[\mathrm{m}]==$ Length $[$ Transpose $[\mathrm{m}]] \& \&$

Complement $[$ Flatten $[$ Chop $[\mathrm{m}]],\{0\}]==\{\}$

End []

EndPackage []

\section{References}

[1] See for example P. Langacker, Phys. Rep. 72, No. 4 (1981) 185-385

[2] F. del Aguila and M. Zrałek, Nucl. Phys. B447, 211 (1995)

[3] J. Bernabéu and P. Pascual, Nucl. Phys. B228, 21 (1983)

[4] S. Wolfram, Mathematica, a System for Doing Mathematics by Computer (AddisonWesley Publishing Company, Redwood City, California, 1988)

[5] F. R. Gantmacher, Théorie des matrices (Dunod, Paris, 1966) 